\title{
Uso del catálogo en línea de la Universidad de Santiago de Chile: estudio estadístico
}

\author{
Ariel Rementeria Piñones \\ Bibliotecario de la Facultad Tecnológica \\ Universidad de Santiago de Chile \\ E-mail aremente@lauca.usach.cl
}

\section{RESUMEN}

Análisis del uso del catálogo en línea de acceso al público (OPAC) en el Sistema de Bibliotecas de la Universidad de Santiago de Chile (USACH). Se utilizó la estadística básica para estudiar el proceso de búsqueda de información en el OPAC, y la metodología del Análisis de las Transacciones, realizadas sobre una muestra probabilística de datos, que representa las búsquedas de los usuarios en los equipos computacionales del campus universitario. Las opciones de búsquedas del OPAC que resultaron más utilizadas por los usuarios fueron: Autor, con una tasa de éxito de 64,6\%, Materia, con una tasa de éxito de $62,4 \%$, y Título, con una tasa de éxito de $60,9 \%$. El 87\% del total de las búsquedas fueron realizadas en el OPAC local-ubicado en la Biblioteca Central-y 13\% en el OPAC remoto, el cual es accesible desde la red corporativa de la USACH.

PalabrasClave Catálogosenlínea, Bididecasuriveritarias Frecueniadeuso

\section{USE OF THE ON-LINE CATALOGUE AT THE UNIVERSITY OF SANTIAGO DO CHILE: A STATISTICAL STUDY \\ ARIEL REMENTERIA-PIÑONES}

\begin{abstract}
An analysis of the use of the public-access on-line catalogue (OPAC) of the Library System of the Universidad de Santiago de Chile (USACH). Basic statistical analysis is used to study the information search processes used in OPAC. Transactions effectuated over a probabilistic sample of searches by campus-based users is the methodology employed. The search options most widely employed by users are: Author, yielding a $64.6 \%$ success rate; Subject, yielding a $62.4 \%$ success rate; and Title, which yielded a success rate of $60.9 \%$. Eight-seven percent of the total number of searches were carried out in the local OPAC located in the Central Library and 13\% were realized from remote locations by accessing the corporate network of USACH.

KeyWbros OrlineGatogues Universitylibraies UseFrequency
\end{abstract}

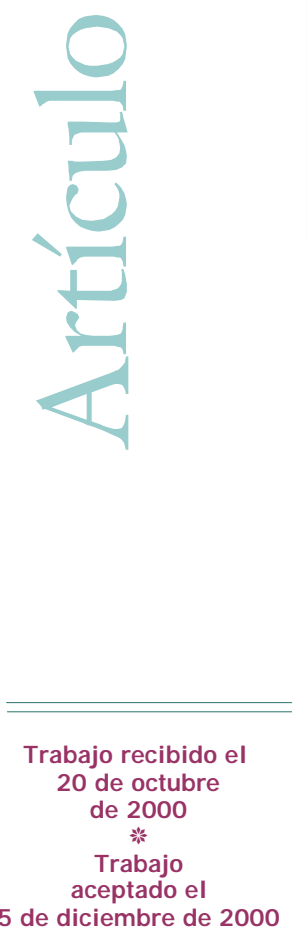

5 de diciembre de 2000 


\section{INTRODUCCIÓN}

Desde hace décadas las bibliotecas universitarias chilenas han ido asimilando las nuevas tecnologías de la información en sus servicios bibliotecarios con el fin de apoyar el estudio y la enseñanza. Los primeros esfuerzos se realizaron en los años setenta, con el poblamiento de bases de datos bibliográficas institucionales y/o cooperativas para el almacenamiento y recuperación de información, y las principales áreas temáticas que se cubrieron fueron: salud, agricultura y economía, desarrollando para ello bases de datos en administradores de archivos, preferentemente en CDS/Microisis; el catálogo colectivo de revistas de la Universidad de Chile fue uno de los primeros catálogos desarrollados con saftuarepropio de la institución. ${ }^{1}$

Más adelante, en la década de los años 80, el Sistema de Bibliotecas de la Pontificia Universidad Católica de Chile desarrolló el primer sattwarenacional para la automatización de bibliotecas. En esta misma época nació la Red Nacional de Información Bibliográfica (RENIB) con el software integrado para bibliotecas NOTIS como base principal para desarrollar la red. ${ }^{2}$

En los años 90, gran parte de las universidades tradicionales iniciaron el proceso de automatización de las funciones básicas de sus bibliotecas tanto con sistemas desarrollados localmente como con sattuarede tipo comercial. ${ }^{3}$

En la actualidad, con el fin de auxiliar a los estudiantes y docentes de la enseñanza superior en la búsqueda de información para sus trabajos académicos, se pone a su disposición el acceso, en las bibliotecas universitarias, a catálogos en línea, a bases de datos en línea o en discos compactos, a redes nacionales o internacionales de información, siendo los catálogos en línea uno de los mayores recursos con que hoy contamos para proveer información bibliográfica a los usuarios de las bibliotecas en la modalidad de autoservicio.

Los alumnos de la Universidad de Santiago de Chile (USACH) tienen acceso a la base de datos bibliográfica de la institución a través de los catálogos en línea del sistema Dynix, satwareque está disponible para esta comunidad desde 1994.

Este estudio es el primer intento por conocer la forma en que los usuarios de las bibliotecas de la USACH utilizan el catálogo en línea de acceso al público (OPAC del inglés: On line Public Access Catalog).

\section{REVISIÓN DE LA LITERATURA}

El interés en los estudios sobre las necesidades y el comportamiento de los usuarios de los catálogos está reflejado en la literatura internacional de la bibliotecología y las ciencias de la información. En los Estados Unidos (desde el año 1930 hasta 1981) existen más de 50 estudios sobre el uso y los usuarios de los catálogos tradicionales de tarjetas, y desde 1980 los estudios relativos al uso de los catálogos en línea OPAC han aparecido constantemente en la literatura bibliotecológica. ${ }^{4}$ 
Sin embargo, en Chile no encontramos estudios realizados y publicados sobre el uso del catálogo en línea, a pesar de que existen diferentes sistemas en pleno funcionamiento que apoyan la gestión de bibliotecas, especialmente en las universidades, donde se ha priorizado inicialmente el uso de los catálogos en línea, ${ }^{5}$ En Chile por ejemplo, están funcionando los programas de gestión bibliotecaria: Multilis, Horizonte, Dynix, Werken, NOTIS, Aleph y aplicaciones en MicroIsis, los cuales tienen acceso a la base de datos a través del OPAC y también poseen funciones estadísticas básicas de las diferentes actividades del sistema.

Las metodologías usadas en los diferentes tipos de estudios han sido: la aplicación de cuestionarios, el análisis de transacción computacional, las observaciones de los usuarios, las entrevistas a individuos o a grupos escogidos, el análisis de los registros del préstamo interbibliotecario (PIB), y las posibles combinaciones de estos métodos. En este estudio se utilizó el análisis de transacción computacional y la estadística básica para administración y ciencias sociales.

\section{METODOLOGÍA}

El Sistema de Bibliotecas de la USACH, compuesto por una Biblioteca Central y 20 Bibliotecas de Especialidad, ofrece sus servicios a 18,000 alumnos, 1,200 funcionarios y 1,400 docentes. En la Biblioteca Central se utiliza el satwarede gestión de bibliotecas Dynix, el cual actúa como nodo principal, y en 3 Bibliotecas de Especialidad funcionan los módulos de Circulación y Catálogo al Público (OPAC).

El módulo de Catálogo en Línea de Acceso al Público (OPAC) de la USACH, es accesible en forma local en la Biblioteca Central y el acceso remoto, desde cualquier equipo de la red corporativa.

El objetivo principal de nuestra investigación es conocer el uso del OPAC en la USACH. Las interrogantes que quisimos responder con este estudio son: 1) ¿dónde utilizan más el catálogo en línea los usuarios de las bibliotecas de la USACH? 2) ¿qué opciones son las más utilizadas por los usuarios? 3) ¿qué tipos de errores cometen frecuentemente los usuarios? 4) ¿cómo buscan o qué patrones de búsqueda utilizan más los usuarios? y 5) ¿qué porcentaje o tasa de aciertos tienen los usuarios del OPAC USACH?

Puesto que éste es un estudio estadístico, se proporcionan los parámetros y estadígrafos más conocidos de los datos, cuya finalidad es aportar herramientas válidas en la posible toma de decisiones relacionada con el catálogo automatizado de la Universidad.

El proceso a estudiar son las consultas o búsquedas de información bibliográfica que hacen los usuarios en el OPAC-USACH, a las cuales denominamos transacción. Para nuestro trabajo, una transacción consiste en la secuencia de caracteres que definen una consulta o búsqueda de información bibliográfica realizada por el usuario en un equipo computacional, la cual es respondida por el sistema. Asimismo, definimos 
análisis de transacción computacional al examen sistemático y detallado de cada instrucción de la búsqueda o consulta hecha por un usuario y la consecuente respuesta de la base de datos como resultado o salida del catálogo en línea. ${ }^{6}$

$\mathrm{El}$ análisis de transacción computacional es utilizado para estudiar los errores, es decir, para determinar las probables causas de las fallas de los usuarios, aunque este tipo de análisis nada nos dice sobre el comportamiento del usuario. El análisis de transacción también es usado como herramienta para la administración del desarrollo de colecciones, para determinar patrones de búsquedas, para efectuar cambios o modificaciones en el sistema y para mejorar o modificar los programas de instrucción o educación de los usuarios. ${ }^{7}$

El presente estudio está dividido en dos partes. La primera es un análisis a los datos que almacena y que provee Dynix (sistema que guarda una cantidad importante de datos como una de las funciones estadísticas de los procesos que controla y ejecuta), y en la segunda parte se analiza una muestra aleatoria de las transacciones del OPAC local de la USACH.

Para este estudio se utilizaron las funciones estadísticas del módulo del catálogo en línea OPAC; una de ellas, es el almacenamiento y contabilización de las transacciones realizadas en el OPAC, las cuales son presentadas en un informe numérico anual o mensual de manera automática automática, por lo que no se requiere dar instrucciones especiales al sistema. A manera de ejemplo, a continuación se presenta un informe originado por el sistema estadístico de Dynix, cuyos datos corresponden a las transacciones realizadas durante el mes de agosto de 1998 en el OPAC local de la USACH.

Reporte 1

Informe Estadístico de las Búsquedas en Dynix. Estadísticas de Búsqueda

Reporte Mensual

RAMA - - BTCA. CENTRAL -

01 AGO 1998

**PUBLIC**

Índice

Búsqueda Alfabética por Autor

Número Decimal Dewey

Búsqueda General por Palabra Clave

Búsqueda Alfabética por Materia

Palabra Clave Autoridad de MATERIA

Búsqueda Alfabética por Serie

Búsqueda Alfabética por Título

Palabra Clave en el Título
Número de Búsquedas

11644

135

690

10997

1216

434

6811

984 
Otra característica del sistema Dynix es que puede hacer un rastreo o seguimiento de todas las transacciones realizadas en el OPAC, ya que almacena la consulta (secuencia de caracteres) y el resultado (la respuesta del sistema), y presenta los datos en un balance numérico - para obtener este tipo de informes hay que dar las instrucciones de inicio y término del rastreo en el sistema, ya que ésta función utiliza importantes recursos computacionales.

A continuación se presenta un ejemplo parcial del informe de rastreo de búsqueda, correspondiente a la opción de búsqueda por Autor, en el OPAC local del 24 de noviembre de 1998.

Reporte 2

Informe Estadístico del Rastreo de Búsquedas en Dynix

*** REPORTE DE RASTREO DE BUSQUEDA *** Iniciando 24 NOV

1998 Impreso 01 Dic. 1998

\begin{tabular}{|c|c|c|c|c|c|c|}
\hline SOURCE & TYPE & TEXT OF SEARCH & HITS & BIB & MRC & COPY \\
\hline PUBLIC & AA & Allende & 4 & 38 & & 2 \\
\hline PUBLIC & AA & McMurry & 1 & 1 & & 0 \\
\hline PUBLIC & AA & Miller & 5 & 3 & & 1 \\
\hline PUBLIC & AA & Midovic & 0 & 0 & & 0 \\
\hline PUBLIC & AA & Zegarra & 1 & 1 & & 1 \\
\hline PUBLIC & AA & Tarquin & 2 & 2 & & 1 \\
\hline PUBLIC & AA & Swokowsky & 2 & 2 & & 2 \\
\hline PUBLIC & AA & Ivanisevich & 3 & 3 & & 2 \\
\hline PUBLIC & AA & Fresco Juan & 1 & 4 & & 0 \\
\hline PUBLIC & AA & Kotler & 2 & 2 & & 3 \\
\hline PUBLIC & AA & Sears & 2 & 2 & & 1 \\
\hline PUBLIC & AA & Alonso & 3 & 4 & & 1 \\
\hline PUBLIC & AA & Benitez & 6 & 7 & & 1 \\
\hline PUBLIC & AA & Valdes & 1 & 1 & & 1 \\
\hline Subtotal & AA & & 33 & 70 & & 16 \\
\hline
\end{tabular}

En la tabulación, cálculo, análisis y presentación de los datos se utilizó la planilla electrónica Excel.

Para responder las interrogantes: 1) ¿dónde utilizan más el catálogo en línea los usuarios de las bibliotecas de la USACH y 2) ¿qué opciones son las más utilizadas por los usuarios? se analizaron los datos almacenados en Dynix de 1995 a 1998, y luego, se obtuvieron las cifras comparativas de uso entre el OPAC local y el remoto.

Para responder las preguntas 3) ¿qué tipos de errores cometen frecuentemente los usuarios? 4) ¿cómo buscan o qué patrones de búsquedas utilizan más los usuarios? y 5) qué porcentaje o tasa de aciertos tienen los usuarios del OPAC-USACH, se llevó a cabo el análisis de las observaciones a través de un muestreo aleatorio simple de las transacciones realizadas en el OPAC local durante el periodo enerodiciembre de 1998.

En la Biblioteca Central los equipos dedicados a la consulta del OPAC local son 11 terminales IBM 3151 con teclados en español, y, para la consulta del OPAC remoto, se dispone de 19 licencias o puertos de comunicación que pueden ser accesibles 
por un servidor o computadora personal conectada a la red corporativa de la USACH. En total, pueden consultar 30 usuarios, en forma simultánea, el OPAC- USACH.

\section{UNIVERSO, SELECCIÓN Y TAMAÑO DE LA MUESTRA.}

Con el fin de determinar qué OPAC era más usado; el local o el remoto, se consideró el universo de datos almacenados en el OPAC de 1995 a 1998 (Ver Cuadro2), por lo que el nivel de confiabilidad ${ }^{8}$ de los resultados para este primer estudio fue del 100\%. Con el análisis de este universo dimos respuesta a las preguntas: 1) ¿dónde utilizan más el catálogo en línea los usuarios de las bibliotecas de la USACH? y 2) ¿qué opciones son las más utilizadas por los usuarios?

Lo anterior representa la primera parte del estudio, el cual pudo realizarse gracias a la disponibilidad tecnológica, y a que la obtención de los datos desde el sistema Dynix fue a un costo y tiempo razonables.

Para realizar la segunda parte del estudio -el análisis de transacciones computacional en el OPAC local-se decidió tomar una muestra aleatoria de 211 horas, de un total de 2,520 horas de atención del OPAC local durante 1998. Para hacer las inferencias estadísticas, de la segunda parte del estudio, la muestra debía ser probabilística, es decir, todas las unidades tenían la misma probabilidad de ser escogidas (la clave para seleccionar una muestra es obtener un listado lo más actualizado posible de los elementos de donde se obtendrá la muestra. Por lo general a este listado se le denomina marco muestral). ${ }^{?}$

La formación de la muestra aleatoria se hizo de la siguiente manera: Primero se elaboró un calendario de atención o disponibilidad de servicio del OPAC local de la Biblioteca Central durante 1998 -que sería nuestra "lista"-, con el fin de registrar las observaciones de 42 semanas, entre las que se escogieron 211 horas al azar.

Como la muestra debía ser lo más representativa posible, se decidió realizar las observaciones de los días hábiles, de enero a diciembre de 1998, excepto los sábados. Así, obtuvimos un "universo-objeto" ya que éste no contiene todos los elementos de un universo real. ${ }^{10}$ Acto seguido, se dividió el horario de atención a usuarios del OPAC local en tramos o intervalos de una hora, otorgando a cada tramo un número entero secuencial del 1 al 11 (por ser once las horas que está disponible el OPAC local para los usuarios). Después, con una Tabla de Números Aleatorios ${ }^{11}$ de 2,000 números, se escogió al azar, por cada día hábil, un intervalo representado con un número del 1 al 11. Por ejemplo, al lunes 20 de abril de 1998 le correspondió el número 6, que representa el intervalo de las 14:00 a las 15:00 horas, según se observa en el Cuadro1, en el cual podemos apreciar parte del calendario que se elaboró para hacer la muestra. Cabe aclarar que el muestreo fue sin reemplazo y de población finita. ${ }^{12}$ 
CUADRO 1

Ejemplo de la confección de la lista para realizar la muestra de los datos en el O PAC local de la Universidad de Santiago de Chile

Periodo 1995 a 1998

\begin{tabular}{|c|c|c|c|c|c|c|c|}
\hline Horario & $\begin{array}{l}\text { Número } \\
\text { asignado } \\
\text { del } \\
\text { Intervalo }\end{array}$ & $\begin{array}{l}\text { Lunes } \\
20 / 4 / 98\end{array}$ & $\begin{array}{c}\text { Martes } \\
21 / 4 / 98\end{array}$ & $\begin{array}{l}\text { Miércoles } \\
22 / 4 / 98\end{array}$ & $\begin{array}{c}\text { Jueves } \\
23 / 4 / 98\end{array}$ & $\begin{array}{c}\text { Viernes } \\
24 / 4 / 98\end{array}$ & $\begin{array}{l}\text { Total de } \\
\text { intervalos }\end{array}$ \\
\hline 9:00 a 10:00 & 1 & & & & & & 0 \\
\hline 10:00 a 11:00 & 2 & & & & & & 0 \\
\hline $11: 00$ a $12: 00$ & 3 & & & & & & 0 \\
\hline $12: 00$ a $13: 00$ & 4 & & & & & & 0 \\
\hline $13: 00$ a $14: 00$ & 5 & & & & & $\mathbf{X}$ & 1 \\
\hline $14: 00$ a $15: 00$ & 6 & $\mathbf{X}$ & & & & & 1 \\
\hline $15: 00$ a $16: 00$ & 7 & & & & & & 0 \\
\hline $16: 00$ a $17: 00$ & 8 & & & & $\mathbf{X}$ & & 1 \\
\hline $17: 00$ a $18: 00$ & 9 & & & $\mathbf{X}$ & & & 1 \\
\hline 18:00 a 19:00 & 10 & & $\mathbf{X}$ & & & & 1 \\
\hline $19: 00$ a $20: 00$ & 11 & & & & & & 0 \\
\hline
\end{tabular}

Con la "lista" se logró obtener un marco muestral confiable y con costos mínimos. Se tomaron observaciones del OPAC local con base en intervalos de tiempo de 60 minutos consecutivos. Así, en cada observación seleccionada al azar se encontraría la actividad realizada por los usuarios en el OPAC local durante una hora. El siguiente paso fue determinar el tamaño de la muestra.

\section{DETERMINACIÓN DEL TAMAÑO DE LA MUESTRA}

¿Por qué hacer un muestreo si el sistema Dynix entrega suficiente información estadística sobre la actividad del OPAC? Porque este sistema nada nos dice sobre la tasa de éxito o fracaso de los usuarios, ni sobre los errores que éstos cometen, ni los títulos o autores más solicitados. Por tal motivo, nos vimos en la necesidad de realizar un muestreo.

En el muestreo, la regla general es: entre más grande sea la muestra, mejor. ${ }^{13}$ Pero ¿cuándo debe ser la muestra grande o cuándo pequeña? Cuando el estudio requiere $100 \%$ de certeza, la muestra debe ser más grande. 
Nosotros decidimos tener un $95 \%$ de nivel de confianza en que los resultados estarían dentro de lo correcto, y aceptar que el 5\% de las veces no lo estarían. (En las ciencias de la información y la bibliotecología, el nivel de confianza generalmente usado es del 95\%). ${ }^{14}$ También decidimos tener un - 5\% de variación en el promedio de la muestra con relación al promedio del universo. Luego de estas decisiones tomamos una muestra experimental o estudio piloto para poder estimar el tamaño de la muestra. ${ }^{15}$

Nuestro ensayo piloto para determinar el tamaño de la muestra consistió en 20 observaciones tentativas escogidas al azar y realizadas en el mes de diciembre de 1997. En la Tabla 1 podemos observar los resultados de este ensayo piloto; y en la Tabla2 el resultado de los cálculos para determinar la muestra, según la Fórmula 1 que se utilizó. ${ }^{16}$ Cada observación corresponde a una hora cronológica en que el OPAC local de la USACH estuvo disponible para los usuarios.

En cada hora observada se contabilizó, por separado, cada una de las cinco opciones de búsqueda que se había decidido estudiar; las demás transacciones no fueron consideradas. De esta forma se logró llegar al resultado de la tabla 1.

Tabla 1

Resumen del Estudio Piloto para Determinar los Tamaños de las Muestras

\begin{tabular}{|c|c|c|c|c|c|c|c|c|c|c|}
\hline $\begin{array}{c}\text { Obser- } \\
\text { vacio- } \\
\text { nes }\end{array}$ & $\begin{array}{c}\text { Autor } \\
\mathbf{x a}\end{array}$ & $\mathbf{X a}^{2}$ & $\begin{array}{c}\text { Materia } \\
\mathbf{x m}\end{array}$ & $\mathbf{x m}^{2}$ & $\begin{array}{c}\text { Título } \\
\mathbf{x t}\end{array}$ & $\mathbf{X t}^{2}$ & $\begin{array}{c}\text { Key } \\
\text { Materia } \\
\mathbf{x k m}\end{array}$ & $\mathbf{x k m}^{2}$ & $\begin{array}{c}\text { Key Tí- } \\
\text { tulo xkt }\end{array}$ & $\mathbf{x k t}^{2}$ \\
\hline 1 & 38 & 1444 & 40 & 1600 & 27 & 729 & 3 & 9 & 2 & 4 \\
\hline 2 & 36 & 1296 & 34 & 1156 & 11 & 121 & 3 & 9 & 2 & 4 \\
\hline 3 & 31 & 961 & 33 & 1089 & 21 & 441 & 7 & 49 & 5 & 25 \\
\hline 4 & 36 & 1296 & 37 & 1369 & 9 & 81 & 2 & 4 & 2 & 4 \\
\hline 5 & 65 & 4225 & 62 & 3844 & 26 & 676 & 3 & 9 & 5 & 25 \\
\hline 6 & 54 & 2916 & 58 & 3364 & 21 & 441 & 4 & 16 & 3 & 9 \\
\hline 7 & 38 & 1444 & 41 & 1681 & 18 & 324 & 5 & 25 & 4 & 16 \\
\hline 8 & 26 & 676 & 26 & 676 & 11 & 121 & 2 & 4 & 2 & 4 \\
\hline 9 & 46 & 2116 & 44 & 1936 & 25 & 625 & 3 & 9 & 4 & 16 \\
\hline 10 & 37 & 1369 & 37 & 1369 & 19 & 361 & 3 & 9 & 3 & 9 \\
\hline 11 & 40 & 1600 & 41 & 1681 & 19 & 361 & 3 & 9 & 5 & 25 \\
\hline 12 & 53 & 2809 & 54 & 2916 & 25 & 625 & 5 & 25 & 2 & 4 \\
\hline 13 & 27 & 729 & 30 & 900 & 12 & 144 & 4 & 16 & 4 & 16 \\
\hline 14 & 55 & 3025 & 56 & 3136 & 25 & 625 & 5 & 25 & 5 & 25 \\
\hline 15 & 57 & 3249 & 60 & 3600 & 27 & 729 & 5 & 25 & 4 & 16 \\
\hline 16 & 59 & 3481 & 60 & 3600 & 28 & 784 & 5 & 25 & 4 & 16 \\
\hline
\end{tabular}


Tabla 1 (cont.)

Resumen del Estudio Piloto para Determinar los Tamaños de las Muestras

\begin{tabular}{|c|c|c|c|c|c|c|c|c|c|c|}
\hline $\begin{array}{c}\text { Obser- } \\
\text { vacio- } \\
\text { nes }\end{array}$ & $\begin{array}{c}\text { Autor } \\
\mathbf{x a}\end{array}$ & $\mathbf{X a}^{2}$ & $\begin{array}{c}\text { Materia } \\
\mathbf{x m}\end{array}$ & $\mathbf{x m}^{2}$ & $\begin{array}{c}\text { Título } \\
\mathbf{x t}\end{array}$ & $\mathbf{X t}^{2}$ & $\begin{array}{c}\text { Key } \\
\mathbf{M a t e r i a} \\
\mathbf{x k m}\end{array}$ & $\mathbf{x k m}^{2}$ & $\begin{array}{c}\text { Key Tí- } \\
\text { tulo xkt }\end{array}$ & $\mathbf{x k t}^{2}$ \\
\hline 17 & 20 & 400 & 31 & 961 & 14 & 196 & 3 & 9 & 2 & 4 \\
\hline 18 & 23 & 529 & 26 & 676 & 14 & 196 & 3 & 9 & 5 & 25 \\
\hline 19 & 21 & 441 & 16 & 256 & 14 & 196 & 4 & 16 & 2 & 4 \\
\hline 20 & 25 & 625 & 22 & 484 & 9 & 81 & 2 & 4 & 3 & 9 \\
\hline & 787 & 34631 & 808 & 36294 & 375 & 7857 & 74 & 306 & 68 & 260 \\
\hline
\end{tabular}

Conforme a los cálculos efectuados con los resultados del estudio piloto, se obtuvieron los siguientes tamaños de muestras para cada opción de búsqueda del OPAC local de la USACH.

Tabla 2

Tamaño de las Muestras según la Prueba Piloto

\begin{tabular}{|c|c|c|c|c|}
\hline Autor xa & Materia xm & Título xt & Key Materia xkm & Key Título xkt \\
\hline 190 & 179 & 188 & 188 & 199 \\
\hline
\end{tabular}

El tamaño de la muestra se obtuvo con la siguiente fórmula:

$$
N^{\prime}=\frac{1600\left[N\left(\sum x^{2}\right)-\left(\sum x\right)^{2}\right]}{\left(\sum x\right)^{2}}
$$

Donde:

$\mathrm{N}^{\prime}=\quad$ Número de observaciones que requiere el criterio de $95 \%$ de confianza y $-5 \%$ de variación.

$\mathrm{N}=\quad$ Número de observaciones efectuadas.

$\sum \mathrm{x}=\quad$ Sumatoria de las observaciones.

$\left(\sum \mathrm{x}\right)^{2}=$ Cuadrado de la sumatoria de las observaciones.

$\sum \mathrm{x}^{2}=$ La sumatoria de los cuadrados de las observaciones.

$1600=$ Cuadrado de la constante usada para este nivel de confianza. 
Resolvamos la fórmula para obtener el tamaño de la muestra para la opción de búsqueda por Autor (Xa):

$$
\begin{gathered}
N^{\prime}=\frac{1600[20(34631)-(619369)]}{(619369)}=\frac{1600[692620-619369]}{619369} \\
N^{\prime}=\frac{1600[73251]}{(619369)}=\frac{117201600}{619369}=189.2
\end{gathered}
$$

$\mathrm{N}^{\prime}=190$ observaciones para la opción Autor (Xa); es práctica común redondear al número entero más cercano.

El estudio piloto determinó que para la opción de búsqueda Autor debíamos tomar 190 observaciones.

En la Tabla2 se puede observar que el tamaño de la muestra menor es 179 y el mayor es 199. Para nuestro estudio decidimos realizar 211 observaciones en total durante 1998 en el OPAC local, de las cuales doce fueron agregadas como extras para poder eliminar posteriormente las que tuvieran algún error.

\section{RESULTADOS Y ANÁLISIS DEL USO DEL CATÁLOGO EN LÍNEA LOCAL Y REMOTO}

En el Cuadro 2 se puede apreciar la incidencia en el uso de cada modalidad del OPAC en un periodo de cuatro años (1995-1998). Cabe señalar que 1995 fue el año de inicio y prueba del sistema.

Vemos que la proporción de uso tiende a mantenerse en cada año del periodo analizado, y que los usuarios del OPAC-USACH utilizan con mayor frecuencia el OPAC local -el cual se encuentra en las dependencias de Biblioteca Central-. Es decir, aproximadamente $87 \%$ de las búsquedas se hacen en el OPAC local, mientras que sólo 13\% se hacen en el OPAC remoto.

Cuadro 2

Cantidad de transacciones realizadas en el catálogo en línea de la Universidad de Santiago de Chile

Periodo 1995 a 1998

\begin{tabular}{|l|r|r|r|r|r|}
\hline \multicolumn{1}{|c|}{ Tipo OPAC } & \multicolumn{1}{c|}{1995} & \multicolumn{1}{c|}{1996} & \multicolumn{1}{c|}{1997} & \multicolumn{1}{c|}{1998} & \multicolumn{1}{c|}{ Total } \\
\hline Búsquedas en el OPAC local & 26601 & 441424 & 370028 & 389129 & 1227182 \\
\hline Búsquedas en el OPAC remoto & 4034 & 63175 & 41545 & 77177 & 185931 \\
\hline Total & 30635 & 504599 & 411573 & 466306 & 1413113 \\
\hline
\end{tabular}


En el aradro3, al analizar las opciones de búsqueda más utilizadas por los usuarios del OPAC-USACH, encontramos que las opciones Autor y Materia, son las preferidas por los usuarios, las cuales representan aproximadamente $71 \%$ de las búsquedas analizadas en cada año.

La opción por Título es la tercera preferida por los usuarios, con $19 \%$ del total de búsquedas aproximadamente. Las opciones Autor, Materia y Título acumularon, en cada periodo analizado, 90\% de las búsquedas realizadas en el OPAC local, mientras que las opciones Palabra clave en la Materia y Palabra clave en el Título solo fueron utilizadas en un 4\% y un 3\% respectivamente. En menor grado de uso están el resto de las opciones, siendo el Número de Clasificación Dewey la opción menos utilizada.

Estos resultados indican que las opciones de búsqueda preferidas por los usuarios se han mantenido constantes en el tiempo estudiado y que todas las opciones de búsqueda del OPAC local son utilizadas. Sin embargo, llama la atención el poco uso que tienen las opciones con Palabra clave como tipo de búsqueda.

Cuadro 3

Distribución porcentual de las opciones de búsqueda más utilizadas en el catálogo en línea de acceso local en la Universidad de Santiago de Chile

Periodo 1995 a 1998

\begin{tabular}{|l|c|c|c|c|}
\hline \multicolumn{1}{|c|}{ O PCIÓ N DE BÚSQ UEDA } & 1995 & 1996 & 1997 & 1998 \\
\hline Autor & 36.2 & 37.3 & 36.6 & 35.3 \\
\hline Materia & 35.1 & 34.6 & 35.2 & 34.7 \\
\hline Título & 18.2 & 19.4 & 19.4 & 19.4 \\
\hline Palabra clave en la Materia & 3.6 & 3.1 & 3.2 & 4.0 \\
\hline Palabra clave en el Título & 3.1 & 2.6 & 2.7 & 3.1 \\
\hline Título de la Serie & 1.6 & 1.3 & 1.4 & 1.6 \\
\hline Palabra clave General & 1.7 & 1.3 & 1.2 & 1.5 \\
\hline $\begin{array}{l}\text { Número de Clasificación } \\
\text { Dewey }\end{array}$ & 0.5 & 0.4 & 0.3 & 0.4 \\
\hline Total \% & 100 & 100 & 100 & 100 \\
\hline
\end{tabular}


En el Cuadro4se pueden observar los resultados del análisis del OPAC remoto.

Cuadro 4

Distribución porcentual de las opciones de búsqueda más utilizadas en el catálogo en línea de acceso remoto en la en la Universidad de Santiago de Chile

Periodo 1995 a 1998

\begin{tabular}{|l|c|c|c|c|}
\hline \multirow{2}{*}{ OPCIÓN DE BÚSQUEDA } & 1995 & 1996 & 1997 & 1998 \\
\hline Autor & 36.9 & 36.2 & 35.8 & 34.8 \\
\hline Materia & 34.4 & 35.0 & 34.9 & 35.4 \\
\hline Título & 18.1 & 19.0 & 19.7 & 18.8 \\
\hline Palabra clave en la Materia & 4.3 & 3.5 & 3.5 & 3.8 \\
\hline Palabra clave en el Título & 3.3 & 3.1 & 2.8 & 3.5 \\
\hline Título de la Serie & 1.1 & 1.4 & 1.5 & 1.6 \\
\hline Palabra clave General & 1.0 & 1.4 & 1.4 & 1.6 \\
\hline Número Clasificación Dewey & 0.9 & 0.4 & 0.4 & 0.5 \\
\hline Total \% & 100 & 100 & 100 & 100 \\
\hline
\end{tabular}

El orden de las preferencias de las opciones de búsqueda se mantuvo igual tanto en el OPAC local como en el remoto, es decir, las tres opciones Autor, Materia y Título acumularon, en cada periodo analizado, $90 \%$ de las búsquedas realizadas en el OPAC remoto resultado simular al de las preferencias de búsqueda en el OPAC local. En la distribución porcentual, la variación entre el OPAC local y el remoto es insignificante para este estudio.

Como en el OPAC local, todas las opciones de búsqueda del OPAC remoto fueron utilizadas, por lo que podemos decir que los usuarios del OPAC-USACH utilizan el OPAC local y el remoto en forma análoga en cuanto a sus opciones de búsqueda.

Aunque la presentación de las opciones de búsqueda tanto para el OPAC local como para el remoto es igual, la diferencia radica en la forma de acceso: el OPAC local es puesto en marcha en la pantalla inicial por personal de la Biblioteca Central, tarea en la que los usuarios no participan. Para accesar y conectarse al OPAC remoto, en cambio, el usuario debe ejecutar la dirección telnet del OPAC. En lo futuro, sería muy conveniente determinar las posibles causas del porqué los usuarios prefieran utilizar el OPAC local al OPAC remoto.

Se utilizó la siguiente fórmula para obtener la media de las transacciones realizadas por hora en 1998. Anteriormente señalamos que el total del tiempo que estuvo 
disponible el OPAC local para los usuarios, fue de 2.529 horas. Por lo tanto, para calcular la media ${ }^{17}$ tenemos:

\section{Fórmula 2}

$$
\mu=\frac{\text { Total de transacciones durante } 1998 \text { en el OPAC local }}{\text { Total de horas disponibles del OPAC local en } 1998}
$$

$$
\mu=\frac{389129}{2529}=153.866
$$

Sin embargo, este promedio o media resultante $(153,9)$ es la suma de los promedios de cada opción de búsqueda, puesto que en la Fórmula 2, en el dividendo (Total de transacciones durante 1998 en OPAC Local), está incluido el total de transacciones de las ocho opciones de búsqueda del OPAC local durante 1998 (Ver Cuadro2), y es conveniente aclarar que para este estudio se analizaron las opciones de búsqueda más utilizadas por los usuarios.

\section{Cuadro 5}

Promedio Mensual de las opciones más usadas del OPAC local de la USACH durante 1998

\begin{tabular}{|l|c|c|c|c|c|c|c|c|c|c|c|c|}
\hline $\begin{array}{l}\text { Opciones más usa- } \\
\text { das en OPAC local } \\
\text { USACH en 1998 }\end{array}$ & Ene. & Mar. & Abr. & May. & Jun. & Jul. & Agos. & Sept. & Oct. & Nov. & Dic. & Media \\
\hline Autor & 48.6 & 41.1 & 88.1 & 66.9 & 60.7 & 60.5 & 42.3 & 75.4 & 40.2 & 31.5 & 42.1 & 54.31 \\
\hline Materia & 48.7 & 35.6 & 84.2 & 68.6 & 64.3 & 63.7 & 40 & 65.6 & 39.6 & 30.9 & 43.4 & 53.15 \\
\hline $\begin{array}{l}\text { Palabra clave en la } \\
\text { Materia }\end{array}$ & 5,1 & 3.6 & 9.7 & 11.7 & 7.3 & 6.9 & 4.4 & 5.8 & 4.1 & 2.9 & 5.3 & 6.15 \\
\hline Título & 24.6 & 26.1 & 50.1 & 38.2 & 33.6 & 31.6 & 24.8 & 37.4 & 21.7 & 16.2 & 21.4 & 29.61 \\
\hline $\begin{array}{l}\text { Palabra clave en el } \\
\text { Título }\end{array}$ & 4.1 & 3.5 & 6.9 & 6.4 & 5.7 & 5.2 & 3.6 & 6.2 & 4.1 & 3.1 & 3.9 & 4.79 \\
\hline Promedios & $\mathbf{1 3 2}$ & $\mathbf{1 0 9 . 9}$ & $\mathbf{2 3 9}$ & $\mathbf{1 9 1 . 8}$ & $\mathbf{1 7 1 . 6}$ & $\mathbf{1 6 7 . 9}$ & $\mathbf{1 1 5 . 1}$ & $\mathbf{1 9 0 . 4}$ & $\mathbf{1 0 9 . 7}$ & $\mathbf{8 4 . 6}$ & $\mathbf{1 1 6 . 1}$ & $\mathbf{1 4 8 . 0 1}$ \\
\hline
\end{tabular}

El aradro5nos muestra el promedio mensual de utilización para cada una de las ocho opciones de búsqueda en el OPAC local de la USACH en 1998.

Si se aplica la Fómula2, se obtiene el promedio de cada una de las ocho opciones, y el divisor de 2.529 se mantiene. 
Como se puede apreciar en el Cuadro5, las opciones más usadas en el OPAC local de la USACH son: Autor, Materia, Título, Palabra clave en la Materia y Palabra clave en el Título, cuyo promedio general es 148,01. Los resultados que aparecen en este cuadro serán comparados más adelante con los de la muestra. Sin embargo, estos datos nada nos dicen sobre el porcentaje o tasa de éxito/fracaso o sobre los errores que cometen los usuarios en sus búsquedas en el OPAC-USACH.

La respuesta a tales interrogantes sólo la pudimos obtener haciendo un muestreo aleatorio de las transacciones del OPAC local.

\section{RESULTADO Y ANÁLISIS DEL MUESTREO DE LAS TRANSACCIONES DEL OPAC LOCAL}

Como ya se señaló, para el estudio del uso a través del análisis de transacciones del OPAC local se consideraron solamente las opciones de búsqueda más utilizadas por los usuarios. Cada búsqueda o consulta realizada por un usuario entre las ocho opciones que ofrece el OPAC local fue considerada como una transacción y por lo tanto pudo ser rastreada y almacenada por el sistema Dynix. Una vez ejecutada la búsqueda por el usuario, éste puede tener aciertos* o no, es decir, éxito o fracaso.

En el Cuadro6podemos apreciar la cantidad de aciertos que se encontraron en la muestra, según la opción más utilizada por los usuarios del OPAC local.

\section{Cuadro 6}

Cantidad de aciertos encontrados en la muestra de las opciones más utilizadas del OPAC local de la Universidad de Santiago de Chile

Periodo enero a diciembre de 1998

\begin{tabular}{|l|c|c|c|c|c|c|}
\hline Tipo de Aciertos & Autor & Materia & Título & $\begin{array}{l}\text { Palabra Clave } \\
\text { en la Materia }\end{array}$ & $\begin{array}{l}\text { Palabra Clave } \\
\text { en el Título }\end{array}$ & Total \\
\hline Sin Acierto & 3885 & 4054 & 2284 & 665 & 560 & 11448 \\
\hline Igual o mayor que 1 & 7089 & 6724 & 3560 & 488 & 438 & 18299 \\
\hline Total & $\mathbf{1 0 9 7 4}$ & $\mathbf{1 0 7 7 8}$ & $\mathbf{5 8 4 4}$ & $\mathbf{1 1 5 3}$ & $\mathbf{9 9 8}$ & $\mathbf{2 9 7 4 7}$ \\
\hline
\end{tabular}

* Definimos como acierto al resultado de igualdad o no-igualdad entre lo que ingresa el usuario como consulta, a través de un equipo computacional, y lo que el sistema entrega o responde. Por lo tanto, éxito o "si acierto", significa que la base de datos bibliográfica a lo menos tiene un registro que contiene lo ingresado por el usuario. El fracaso o "no acierto" significa que lo ingresado por el usuario no está en la base de datos. 
Los 11,448 "no aciertos" significan el 38,5\% de las transacciones de la muestra sin aciertos o sea la tasa de fracaso de la muestra, mientras que el $61,5 \%$ de las transacciones fueron la tasa de éxito de la muestra.

En el Cuadro7aparece cada opción con su propia tasa de éxito/fracaso. Por ejemplo; la opción Autor tiene una tasa de éxito de 64,6\%.

Cuadro 7

Cantidad porcentual de aciertos de la muestra en las opciones más utilizadas del O PAC Iocal de la Universidad de Santiago de Chile

Periodo enero a diciembre de 1998

\begin{tabular}{|l|c|c|c|c|c|}
\hline Tipo de Aciertos & Autor & Materia & Título & $\begin{array}{c}\text { Palabra Clave en } \\
\text { la Materia }\end{array}$ & $\begin{array}{c}\text { Palabra Clave en } \\
\text { el Título }\end{array}$ \\
\hline Sin Acierto & 35.4 & 37.6 & 39.1 & 57.7 & 56.1 \\
\hline Igual o mayor que 1 & $\mathbf{6 4 . 6}$ & $\mathbf{6 2 . 4}$ & $\mathbf{6 0 . 9}$ & 42.3 & 43.9 \\
\hline Total & $100 \%$ & $100 \%$ & $100 \%$ & $100 \%$ & $100 \%$ \\
\hline
\end{tabular}

De las 11.448 transacciones sin aciertos, 8.394 (28,2\% de la muestra) no estaban en la base de datos cuando se realizó la búsqueda o consulta. Las 3.054 (10,3\% de la muestra) transacciones restantes corresponden a errores tipográficos.

Los tipos de errores tipográficos más comunes que encontramos fueron los de escritura. Se contabilizaron 1.717 errores de palabras (5,8\% de la muestra) por falta de algún carácter en su secuencia o por uno o varios caracteres de más.

Cuadro 8

Promedio Mensual de las opciones más usadas del OPAC local de la USACH según muestreo de 1998

\begin{tabular}{|l|c|c|c|c|c|c|c|c|c|c|c|c|}
\hline $\begin{array}{l}\text { Opciones más usadas } \\
\text { en el OPAC local } \\
\text { USACH en 1998 }\end{array}$ & Ene. & Mar. & Abr. & May. & Jun. & Jul. & Agos. & Sept. & Oct. & Nov. & Dic. & Media \\
\hline Autor & 47 & 40 & 87 & 66 & 60 & 59 & 41 & 73 & 38 & 30 & 40 & 52.82 \\
\hline Materia & 48 & 34 & 84 & 67 & 63 & 62 & 38 & 65 & 38 & 30 & 41 & 51.82 \\
\hline $\begin{array}{l}\text { Palabra clave en la } \\
\text { Materia }\end{array}$ & 5 & 3 & 8 & 10 & 6 & 6 & 4 & 5 & 4 & 2 & 5 & 5.27 \\
\hline Título & 25 & 26 & 48 & 37 & 32 & 30 & 23 & 34 & 20 & 15 & 20 & 28.18 \\
\hline $\begin{array}{l}\text { Palabra clave en el Tí- } \\
\text { tulo }\end{array}$ & 4 & 3 & 6 & 5 & 5 & 4 & 3 & 5 & 4 & 2 & 3 & 4 \\
\hline Promedios & $\mathbf{1 2 9}$ & $\mathbf{1 0 6}$ & $\mathbf{2 3 3}$ & $\mathbf{1 8 5}$ & $\mathbf{1 6 6}$ & $\mathbf{1 6 1}$ & $\mathbf{1 0 9}$ & $\mathbf{1 8 2}$ & $\mathbf{1 0 4}$ & $\mathbf{7 9}$ & $\mathbf{1 0 9}$ & $\mathbf{1 4 2 . 0 9}$ \\
\hline
\end{tabular}


Debido al mal manejo del equipo, específicamente del teclado de la terminal, encontramos 1.337 errores (4,5\% de la muestra) en la entrada de caracteres no válidos para el sistema.

Ahora, veamos los promedios específicos para la muestra y comparémoslos con los promedios del universo objeto del Cuadro5.

En el Cuadro8 se pueden observar los promedios mensuales de la muestra.

El promedio general de la muestra es 142,09 y la variación que aceptamos del promedio muestral es de $-5 \%$. Por lo tanto, multiplicamos 142,09 por $5 \%$, y obtenemos 7,1, lo que significa que el promedio general del universo está entre 142,09 menos 7,1 y 142,09 más 7,1. Entonces, tenemos que entre 134,99 y 149,19 debe estar nuestro promedio general, el cual sabemos que es 148,01 con lo que demostramos que los cálculos estadísticos aplicados fueron los correctos, al menos en relación con el promedio general. En el Cuadro9 aparece la comparación de los promedios de la muestra y los del universo objeto.

Cuadro 9

Comparación de promedios entre la Muestra y el Universo

\begin{tabular}{|l|c|c|c|}
\hline \multicolumn{1}{|c|}{ Medida Descriptiva } & Muestra & Universo & \% de Variación \\
\hline Promedio General & 142,09 & 148,01 & 4,16 \\
\hline Promedio Autor & 52,82 & 54,31 & 2,82 \\
\hline Promedio Materia & 51,82 & 53,15 & 2,56 \\
\hline Promedio Palabra Clave Materia & 5,27 & 6,15 & 16,69 \\
\hline Promedio Título & 28,18 & 29,61 & 5,07 \\
\hline Promedio Palabra Clave Título & 4,00 & 4,79 & 19,75 \\
\hline
\end{tabular}

Con relación a la variación de $-5 \%$ que decidimos aceptar entre el promedio de la muestra y el universo, podemos asegurar que ésta se cumplió en los promedios de las opciones de Autor, Materia y Título, resultando ser las opciones de búsqueda más utilizadas por los usuarios del OPAC-USACH.

Los promedios de la muestra de las opciones Palabra Clave de Materia y Palabra Clave de Título, tienen una variación superior a $-5 \%$ que decidimos aceptar. Esta variación fue de 16\% y 20\% respectivamente, es decir, el tamaño de la muestra para estas dos opciones de búsquedas fue insuficiente; debió ser mayor, puesto que la magnitud de tal variación no es aceptable en los estudios de bibliotecología.

Por lo anterior, podemos decir que los resultados de nuestro estudio para estas dos opciones de búsqueda tienen un porcentaje promedio de error de $18 \%$, por lo que no podemos obtener conclusiones muy válidas. 


\section{CONCLUSIONES}

No existen estudios publicados en cuanto al uso de catálogos en línea relacionados con la experiencia chilena, en contraste con la cantidad de saftuarede gestión bibliotecaria instaladas y funcionando en el país, especialmente en las bibliotecas universitarias.

El catálogo en línea base de nuestro estudio, es un medio de comunicación entre el usuario y la base de datos bibliográfica, y se analizó como un elemento que está involucrado en el proceso de comunicación de la información bibliográfica.

El estudio del uso del catálogo en línea de la USACH nos confirmó que la búsqueda de información es un proceso complejo y que los usuarios de este catálogo son muy heterogéneos en cuanto a sus necesidades de información, su habilidad en la búsqueda de información, y su experiencia y conocimientos sobre el equipo computacional.

El análisis de transacción computacional utilizado para este estudio, nos proporcionó los elementos necesarios para responder a nuestras interrogantes sobre el uso del OPAC-USACH, y pensamos que instituciones similares a la nuestra, que tengan autorizado su catálogo al público, pueden adoptar este análisis ajustándolo a sus requerimientos.

Los resultados del análisis de las transacciones nos revelaron lo siguiente: que existen usuarios del OPAC de la USACH que experimentan dificultades con las opciones básicas de búsqueda de información bibliográfica en este sistema.

* Que las transacciones realizadas por los usuarios en el catálogo en línea pueden ser estudiadas y analizadas para hacer las modificaciones pertinentes al sistema.

* Que los usuarios de la USACH utilizan en mayor o menor grado todas las opciones de búsquedas del OPAC.

* Que los usuarios utilizan el OPAC, local y remoto, en forma muy similar en cuanto a sus preferencias de opciones de búsqueda.

* Que porcentaje de utilización del OPAC local (ubicado en Biblioteca Central de la USACH) mayor que el del OPAC remoto: $87 \%$ y $13 \%$ respectivamente.

* Que el tipo de búsqueda por Autor, Materia y Título son las opciones preferidas por los usuarios del catálogo en línea de la USACH. Cuantitativamente podemos decir que por cada 100 consultas realizadas en este catálogo, 36 son búsquedas por Autor; 35, por Materia; 19, por Título; 4, por Palabra clave en la Materia; 3, por Palabra clave en el Título; 1, por Titulo de la Serie; 1, por Palabra clave General; y 1 por Número Clasificación Dewey.

Para el tamaño y toma de la muestra, se utilizó un modelo válido solamente para las opciones de búsqueda de mayor uso del OPAC USACH: Autor, Materia y Título

Los parámetros del universo para 1998 en el OPAC local fueron los siguientes:

Promedio general 153,9. Es decir, en el OPAC local de la USACH se realizaron en promedio 153,9 transacciones por hora cronológica. 
Los promedios específicos de las cinco opciones más usadas fueron;

* Autor: 54,31 transacciones por hora.

* Materia: 53,15 transacciones por hora.

* Título: 29,61 transacciones por hora.

* Palabra clave en la Materia: 6,15 transacciones por hora.

* Palabra clave en el Título: 4,79 transacciones por hora.

La muestra de 211 horas, representa 8,34\% de las 2.529 horas que el OPAC local estuvo disponible, cuyo análisis nos permitió hacer las siguientes inferencias sobre el uso del OPAC para las opciones de búsqueda por Autor, Materia y Título:

* El 62\% de las transacciones tiene uno o más aciertos;

* El 28\% de las transacciones no está en la base de datos;

* El 10\% de las transacciones tiene errores tipográficos;

Lo anterior es válido para 90\% de las transacciones, con una certeza del $95 \%$ de que los resultados son los correctos.

Se pudo establecer la tasa de éxito/fracaso de las siguientes opciones de búsqueda en el OPAC local de la USACH:

* Autor: éxito, 64.6\%.

* Materia: éxito, $62.4 \%$.

* Título: éxito, $60.9 \%$.

Estas opciones corresponden a 90\% de las transacciones del OPAC local de la USACH y las tasas de éxito/fracaso tienen una certeza de $95 \%$.

Para determinar la tasa de éxito/fracaso de las búsquedas o para establecer los tipos de errores que cometen los usuarios, tuvimos que realizar estudios basándonos en muestras probabilísticas para llegar a resultados confiables ya que el sistema de gestión de bibliotecas Dynix sólo entrega información general de la actividad del OPAC, con los datos y herramientas básicos para este tipo de etudios.

Por otro lado, se desconoce si los usuarios del OPAC USACH utilizan o no las pantallas de ayuda puesto que no existe en el sistema Dynix una herramienta que nos ayude a responder ésta interrogante.

\section{NOTAS BIBLIOGRÁFICAS}

1. Seminario sobre Automatización de Bibliotecas Universitarias. Conclusiones. Santiago, 28 y 29 de julio de 1997 organizado por el Centro de Capacitación en Información CECAPI, Universidad de Chile. p. 1.

2. Ibid

3. Ilid p. 2.

4. Connaway, L.S.; Johnson, D.W.; Searing, S.E. Onlinecatalogsfromtheuse'spespeetive theuseaffoasgrapinteviens Colleges \& Research Libraries, 58(5)403-420, september 1997.

5. Seminario sobre Automatización de Bibliotecas Universitarias. Op at, p. 3 
6. Blecic, D.D.; Bangalore, N.S.; Dorsch, J.L. \&al. Usingtransactionloganalysistoim provePAC renieval reults Colleges \& Research Libraries, 59(1)39-50, january 1998.

7. Ibid p. 40.

8. Hernon, Peter. Detemination ofsample, sizeandsdetion of thesample concepts, general saures, andsoftuare Colleges \& Research Libraries, 55(2)171-179, March, 1994.

9. Berenson, Mark L. Estadísticabásica enadministradón, comeqtosyaplicadiones 4a.ed. México, Prentice-Hall Hispanoamericana, 1992. p. 19.

10. Ihid

11. Freund, John; Frank William. Elementos modemos de estadística empresanial. Colombia, Prentice Hall Internacional, 1973.

12. Berenson, Mark L. Estadística básica enadministradón, conęptosyaplicaciones Op at, p. 19.

13. Hernon, Peter. Deteminationofsample, sizeandsdetion ofthesample concepts, general soures, andsoftware Op it, p. 173.

14. Ibid p. 174.

15. Berenson, Mark L. Estadística básica enadministración coneqtosyaplicaciones Op at. p. 345.

16. Dougherty, Richard; Heinritz, Fred. Sampling. En: Lubans, John; Chapman, Edward. Readerinlibrarysystensanalysis Englewood, Microcard Edition Book, 1975. pp. 123-144.

17. Berenson, Mark L. Estadística básica enadministradón, coneqptosyaplicadiones Op at. p. 71.

\section{BIBLIOGRAFÍA CONSULTADA}

Cuevas S., Claudia y otros. Aplicaciones de conceptodehipetextoalos catálogosenlínea Seminario para optar al título de Bibliotecario Documentalista. Instituto Profesional de Santiago, Escuela de Bibliotecología, Santiago de Chile, 1989.

León U., Norma; Piñol G., Flora; Sánchez S., Ximena. Dełeciónde neceidades deinfomaión dela commidad acadénica dilena Comisión Asesora de Bibliotecas y Documentación (CABID) Consejo de Rectores; UNESCO/PGI, Santiago, 1992. 